\title{
Inflammatory-associated obstructions of the male reproductive tract
}

\author{
G. R. Dohle \\ Department of Urology, Erasmus University Medical Center, Rotterdam, The Netherlands
}

Key words. Male genital obstruction-male infertility - urogenital infection

\begin{abstract}
Summary. A history of urogenital inflammation occurs in $5-12 \%$ of men attending infertility clinics. Usually, infection has a detrimental effect on sperm quality by reducing concentration and motility, and possibly affecting the number of morphological normal spermatozoa. In addition, infection may be the source of auto-antibodies against spermatozoa, found in about $8 \%$ of the infertile male population. In contrast to the situation in women, there is no clear evidence that male accessory gland infections can result in epididymal blockage or vassal obstruction, with the exception of genital tuberculosis. Although Chlamydia trachomatis is a well-documented source of chronic prostatitis, the infection does not seem to cause obstruction of the reproductive tract, as it does in women. If male urogenital infection causes obstruction it is most likely located at the level of the ejaculatory ducts. Chronic prostatitis has been proved to cause scarring of the prostatic and ejaculatory ducts, resulting in low seminal volume with low fructose and alpha-glucosidase. Many of these men present with severe oligozoospermia or azoospermia, normal size testis and normal gonadotrophins. We performed an excisional testicular biopsy in all men presenting with $<1$ million spermatozoa per millilitre and found that 39 of $78(50 \%)$ had a normal spermatogenesis. A history of male accessory genital infection was found in $12 \%$ of the men and $10 \%$ had abnormalities found on transrectal ultrasound of the prostate (like oedema, dilatation of the seminal vesicles and ejaculatory ducts) intraprostatic calcifications and dilatation of the periprostatic venous plexus. Ejaculatory duct obstruction is a common cause of male infertility and infections are present in
\end{abstract}

Correspondence: G. R. Dohle, MD, PhD, Department of Urology, Erasmus University Medical Cienter Rotterdam, P.O. Box 2040, 3000 CA Rotterdam, The Netherlands. E-mail: g.r.dohle@erasmusmc.nl at least $22-50 \%$ of these men. Transurethral resection of the ejaculatory ducts may result in a significant improvement of the sperm quality and in spontaneous pregnancies in up to $25 \%$ of the couples. In case of failure sperm aspiration from the epididymis and intracytoplasmic sperm injection is the treatment of choice.

\section{Introduction}

Infections of the male genital tract are probably common and usually self-limiting (Purvis \& Christiansen, 1993). The male accessory sex glands often harbour microorganisms (like ureoplasma and Chlamydia trachomatis), but many of the men are asymptomatic. Occasionally, these microorganisms cause symptomatic prostato-vesiculitis with deterioration of the semen quality and leucospermia. Impairment of sperm function and the formation of an excess of reactive oxygen species (ROS) may be found. The deleterious effect of ROS on semen quality has been documented and reviewed (Sharma \& Agarwal, 1996). There are several reasons why spermatozoa are more vulnerable to ROS than other cells. First, the sperm membrane contains a high level of polyunsaturated fatty acids, which are extremely susceptible to peroxidation. Peroxidative damage may result in a loss of membrane functions and may lead to a reduction in fertilizing ability, motility and viability. Secondly, in contrast to other cells, spermatozoa have a very limited system to repair damaged structures as a consequence of their small amount of cytoplasm and an inactive, highly condensed chromatin. Thirdly, spermatozoa are equipped with a poor defence system against ROS, as catalase is fully absent, and glutathione peroxidase and superoxide dismutase are present in relatively low amounts. 
Chronic infection of the male accessory glands may result in scarring and obstruction of the ejaculatory ducts (EDOs) (Goluboff et al., 1995). Usually, these obstructions are incomplete, causing poor sperm quality with low seminal volume. This diagnosis can be suspected in men with severe oligozoospermia or azoospermia, normal physical findings and normal serum follicle-stimulating hormone (FSH) (Belmonte \& Martin de Serrano, 1998). Other features that suggest obstruction are epididymal congestion, enlarged seminal vesicles and cystic lesions of the epididymis and prostate (Kim et al., 1997; Pierik et al., 1999).

In order to determine the true incidence of partial obstruction of the male reproductive tract in oligozoospermia and to offer the option of surgical exploration and microsurgical reconstruction, a testicular biopsy was performed in 78 patients with $<1$ million spermatozoa per millilitre and the results of the testicular histology were analysed in relation to the medical history, the physical findings and the FSH. In addition, the results of microsurgical repair of the seminal tract in men with normal spermatogenesis and the results of intracytoplasmic sperm injection (ICSI) were determined in these patients. Furthermore, the literature on male accessory gland infection and obstruction of the male reproductive tract were analysed in this study.

\section{Materials and methods}

Men with a sperm concentration of $<1 \times 10^{6}$ spermatozoa per millilitre were investigated for possible partial obstruction, by performing a testicular biopsy under local anaesthesia. Spermatogenesis was determined by Johnsen's (1970) scoring method. A testicular biopsy was performed in 78 men with severe oligozoospermia.

Andrological investigations included history taking, physical examination, semen analysis and gonadotrophins. In men with a seminal volume $<1.0 \mathrm{~cm}^{3}$ a transrectal ultrasound (TRUS) investigation of the prostate and the seminal vesicles was performed.

Treatment options included scrotal exploration with vasography, vaso-epididymostomy and epididymal sperm aspiration- for ICSI. In men with EDO a transurethral resection of the ejaculatory ducts (TURED) was offered. TURED was performed under local anaesthesia with the patient in lithotomy position. The ducts were incised next to the verumontanum under TRUS guidance and under direct vision through a Collins hook (Karl Storz GmbH and Co., Tuttingen, Germany). Generally, the prostatic floor was incised between the bladder neck and the verumontanum, which resulted in complete opening of the ejaculatory ducts. Minimal coagulation was used. A Ch 16 transurethral catheter (Bard, Crawley, UK) was introduced in the bladder and removed 24-h postoperatively.

Alternatively, in couples who preferred artificial reproductive techniques, ICSI was offered if motile spermatozoa were found in the ejaculate. Statistical analysis of the data was performed using the nonparametric Student's $t$-test, the Wilcoxon rank test and Spearman's correlation procedure, where appropriate. Two-sided $P$-values $<0.05$ were considered significant.

\section{Results}

The medical history revealed male accessory gland infection in $12.8 \%$, previous hernia repair in $14.1 \%$ and a history of cryptorchidism in $12.8 \%$. A normal or slightly disturbed spermatogenesis Johnsen score $>8)$ was present in 39 of $78(50 \%)$ men. Hernia repair occurred more often in men with a normal spermatogenesis. A varicocele was predominantly observed in men with a disturbed spermatogenesis. FSH was significantly lower $(P<0.0001)$ in men with normal spermatogenesis. Table 1 summarizes the results of the andrological evaluation of the 78 patients.

Male accessory gland infection was present in 10 men $(12.8 \%)$ and a previous testicular tumour in four $(5.1 \%)$. The mean infertility duration was 2.9 years. TRUS was performed in 10 patients and six were reported as abnormal (four with cystic lesions in the prostate midline and two with dilatation of the seminal vesicles). Scrotal ultrasound was performed in 66 men and showed abnormalities in 10 men (including nine cases of varicocele and one testicular tumour). In men with a normal spermatogenesis only one varicocele was detected by ultrasound versus eight varicoceles in men with impaired spermatogenesis.

\begin{tabular}{|c|c|c|c|}
\hline & Obstruction & No obstruction & Total $(\%)$ \\
\hline $\begin{array}{l}\text { Childhood hernia } \\
\text { repair }\end{array}$ & 8 & 3 & $11(14.1)$ \\
\hline $\begin{array}{l}\text { Cryptorchidism/ } \\
\text { orchidopexia }\end{array}$ & 5 & 5 & $10(12.8)$ \\
\hline $\begin{array}{l}\text { Male accessory } \\
\text { gland infection }\end{array}$ & 5 & 5 & $10(12.8)$ \\
\hline Malignancy & 3 & 4 & $7(8.9)$ \\
\hline Chronic disease & 0 & 3 & $3(3.81)$ \\
\hline Varicocele & 1 & 8 & $9(11.5)$ \\
\hline Total & 22 & $28 \cdots$ & $50 / 78(64.1)$ \\
\hline
\end{tabular}


Transurethral resection of the ejaculatory ducts was performed in two patients and resulted in improved semen quality in one man, but not in a spontaneous pregnancy. A microsurgical repair of the seminal tract (vasoepididymostomy) was performed in nine men and resulted in significantly improved semen quality in four, and spontaneous pregnancy in two couples. Alternatively, ICSI was performed in 24 couples and resulted in four $(16.6 \%)$ ongoing pregnancies.

\section{Discussion}

Acquired ductal obstructions account for most of the cases of obstructive azoospermia. Infections of the ductal system, and surgery of the scrotum and the groin are the most important causes of this type of obstructions. Ductal infections used to be caused by ascending urinary tract infections, gonorrhoea and tuberculosis. Recently, Chlamydia trachomatis has been recognized as an infective agent, possibly causing multiple blocks of the ductal system. Epididymal blocks are often asymmetrical and usually found in the corpus and the tail. A history of epididymitis or urethritis is often found. Coexisting EDO may occur (Jarvi et al., 1998).

For the definite diagnosis of obstruction a testicular biopsy is required, confirming the presence of mature spermatids and spermatozoa in the seminiferous tubules (Silber \& Rodriguez-Rigau, 1981). Because of the invasive nature of this procedure, the limited results of surgical treatment of obstructions of the seminal path and alternative options like assisted reproductive treatments, testicular biopsies are not routinely performed in men with oligozoospermia. However, in selective cases the state of spermatogenesis could be assessed to determine the possibility of performing a microsurgical vasoepididymostomy together with an epididymal sperm aspiration and cryopreservation.

In men with progressive deterioration of semen quality and in men with a low semen volume signs of an EDO can be found on TRUS of the prostate. Both surgical incision of cystic lesions in the prostate and aspiration of spermatozoa from the seminal vesicles are treatment options that may require information about the state of spermatogenesis in advance, as EDOs frequently coincide with obstructions of the epididymis (Berardinucci et al., 1998).

In the past, EDO was diagnosed by vasography (Banner \& Hassler, 1978). A complete block in the ejaculatory ducts is conclusive for the diagnosis of total obstruction. However, the main drawback is the invasiveness of the procedure and the subsequent risk of iatrogenic occlusion. In contrast, TRUS is readily available, inexpensive and noninvasive. The ultrasonographic diagnosis of EDO is based upon dilatation of seminal vesicles or abnormalities such as midline prostatic cysts or calcifications in the region of the ejaculatory ducts. Unfortunately, not all patients with EDO have dilated seminal vesicles and, conversely, not all patients with dilated seminal vesicles have EDO (Jarow, 1993).

TURED has become the standard treatment for EDO (Jarow, 1996). The overall success rate of TURED has been quite good. In the literature $>100$ patients have been described as having had this procedure for subfertility and $50 \%$ showed improvement in semen parameters. Spontaneous pregnancies occurred in $25 \%$ of the cases. Pryor \& Hendry (1991) performed a transurethral incision of a Mullerian cyst in 21 patients, of which $10(48 \%)$ experienced an undisclosed improvement in semen quality and eight $(38 \%)$ partners conceived. Dik et al. (1996), in their series of TURED in patients with prostatitis-like symptoms, found a significant improvement in semen quantity and quality in two of three infertile patients. Moreover, eight of the 10 patients with prostatitis-like symptoms who had pre-operatively small volume ejaculate without infertility demonstrated improvement in semen volume after TURED. These results clearly advocate a functional relationship between EDO and low semen volume. Our results of TURED are disappointing: in a series of 15 patients only $46 \%$ patients demonstrated an improvement in seminal volume and in one patient improvement in sperm concentration was observed. Sperm motility was not affected in any patient. Spontaneous pregnancies did not occur after TURED (Cornel et al., 1999).

\section{Conclusion}

Subtotal obstruction of the male reproductive tract is a frequent cause of severe oligozoospermia in men with a normal testicular volume and a normal FSH. In other cases, an epididymal dysfunction might explain the oligozoospermia in men with a normal testicular biopsy score. Patients with subfertility based only on low semen volume might benefit from improvement in seminal volume, because the concomitant increase in $\mathrm{pH}$ of the seminal fluid may protect the spermatozoa against the harmful influence of the acid vaginal mucous. Microsurgical repair and epididymal sperm aspiration for cryopreservation should be considered in men with epididymal obstruction after urogenital infection. In case of failed surgical treatment ICSI can be performed with surgically retrieved spermatozoa. 


\section{References}

Banner MP, Hassler R (1978) The normal vesiculogram. Radiology 128:339-344.

Belmonte IG, Martin de Serrano MN (1998) Partial obstruction of the seminal path, a frequent cause of oligozoospermia in men. Hum Reprod 13:3402-3405.

Berardinucci D, Zini A, Jarvi K (1998) Microsurgical reconstruction for epididymal obstruction. J Urol 159:831834.

Cornel EB, Dohle GR, Meuleman EJ (1999) Transurethral deroofing of midline prostatic cysts for subfertile men. Hum Reprod 14:2297-2300.

Dik P, Lock TM, Schrier BP, Zeijlemaker BY, Boon TA (1996) Transurethral marsupialization of a medial prostatic cyst in patients with prostatitis-like symptoms. J Urol 155:13011304.

Goluboff ET, Stifelman MD, Fisch H (1995) Ejaculatory duct obstruction in infertile men. Urology 45:925-931.

Jarow JP (1993) Transrectal ultrasonography in the infertile man. Fertil Steril 60:1035-1039.

Jarow JP (1996) Diagnosis and management of ejaculatory duct obstruction. Tech Urol 2:79-85.

Jarvi K, Zini A, Buckspan MB, Asch M, Ginzburg B, Margolis M (1998) Adverse effects on vasoepididymostomy outcomes for men with concomitant abnormalities in the prostate and seminal vesicle. J Urol 160:1410-1412.

Johnsen SG (1970) Testicular biopsy score count - a method for registration of spermatogenesis in human testis: normal values and results in 335 hypogonadal males. Hormones $1: 1-24$.

Kim ED, Onel E, Honing SC (1997) The prevalence of cystic abnormalities of the prostate involving the ejaculatory ducts as detected by transrectal ultrasound. Int Urol Nephrol 29:647-652.

Pierik FH, Dohle GR, van Muiswinkel JM, Vreeburg JT, Weber RF (1999) Is routine scrotal ultrasound advantageous in infertile men? J Urol 162:1618-1620.

Pryor JP, Hendry WF (1991) Ejaculatory duct obstruction in subfertile males: analysis of 87 patients. Fertil Steril 56: $725-730$

Purvis K, Christiansen E (1993) Infection of the male reproductive tract. Impact, diagnosis and treatment in relation to male infertility. Int J Androl 16:1-13.

Sharma R, Agarwal A (1996) Role of reactive oxygen species in male infertility. Urology 48:835-850.

Silber SJ, Rodriguez-Rigau LJ (1981) Quantitative analysis of testicle biopsy: determinations of partial obstruction and prediction of sperm count after surgery for obstruction. Fert Steril 36:480-485. 